\title{
Alteraciones lingüisticas en la enfermedad de Parkinson. Aproximación estadística a un estudio con variantes
}

\author{
María Jesús Paredes Duartes \\ Universidad de Cádiz \\ Facultad de Filosofia y Letras \\ Edificio Simón Bolivar, $2^{\mathrm{a}}$ planta \\ Plaza Simón Bolívar, s/n \\ 11002 Cádiz \\ E-mail: mariajesus.paredes@uca.es
}

\author{
RAÚl EsPINOSA Rosso \\ Hospital Universitario Puerta del Mar \\ Unidad de Gestión Clínica de Neurología \\ Avenida Ana de Viya, 21 \\ 11009 Cádiz \\ E-mail: raulespinosarosso@gmail.es
}

\section{ALTERACIONES LINGÜÍSTICAS EN LA ENFERMEDAD DE PAR- KINSON. APROXIMACIÓN ES- TADÍSTICA A UN ESTUDIO CON VARIANTES}

\begin{abstract}
RESUMEN: No existen demasiados estudios sobre las alteraciones del lenguaje en enfermos de párkinson y los que hay no ofrecen una perspectiva completa de cómo están afectados los diferentes componentes del lenguaje (fonético-fonológico, gramatical, léxico, semántico, textual y pragmático) que se localizan en distintas zonas cerebrales. Nuestro objetivo es estudiar la relación entre las alteraciones del lenguaje y diversos aspectos clínicos y sociodemográficos en un grupo de pacientes afectados de párkinson. Hemos seleccionado 46 , de los cuales 42 presentan alteraciones lingüisticas de diversa indole que hemos relacionado con variables sociodemográficas (edad, sexo y nivel cultural) y clínicas (tiempo de evolución de la enfermedad, escala Hoeh\&Yahr, UPDRS-III y la dosis de L-dopa). Según la distribución de las variables, se ha utilizado el test T de Student o U de Mann Withney.
\end{abstract}

PALABRAS Claves: Lingüística clínica; alteraciones lingüísticas; Enfermedad de Parkinson; variables clinicas; variables sociodemográficas.

SUMARIO: 1. Introducción. 2. Material y método. 3. Resultados. 4. Discusión. 5. Conclusiones. 6. Limitaciones del estudio.

\section{LINGUISTIC DISORDER IN PARKINSON'S DISEASE. STATISTICAL APPROACH TO A STUDY WITH VARIANTS}

ABSTRACT: There are not many studies on linguistic disturbances of Parkinson patients and a few we can find do not offer a complete picture about how all the language components are affected (phonetic-phonologic, grammatical, lexical, semantic, pragmatic and textual level), which are located in different brain areas.

Our main aim has to do with the relationship between language alterations and several clinical facts, as well as sociodemographic aspects within a group of patients who are affected by Parkinson. We have selected 46 patients, 42 of them have different kind of language disturbances, which have been related with socio-demographic (age, sex and cultural level) and clinical variables (progression time of the disease, Hoeh \& Yahr Scale, UPDRS-III, and L-dopa treatment). According to the distributions of previous variables, Test $\mathrm{T}$ of Student or U of Mann Withney has been used.

KEY WORDS: Clinical Linguistic; linguistic disorders; Parkinson disease; clinical variables; socio-demographic variants.

SUMMARY: 1 . Introduction. 2 . Material and method. 3. Results. 4. Discussion. 5. Conclusions. 6. Study limitations.

\section{MODIFICATIONS LINGUISTIQUES DE LA MALADIE DE PARKINSON. APPROCHE STATISTIQUE D'UNE ETUDE AVEC VARIANTES}

RÉSUMÉ: Il n'y a pas beaucoup d'études sur les altérations du langage chez les patients atteints de Parkinson et celles qui existent, n'offrent pas une perspective complète de la façon dont les différentes composantes du langage, qui se trouvent dans des zones différentes du cerveau, sont affectées (phonétique-phonologique, grammaticale, lexicale, sémantique, textuelle et pragmatique). Notre objectif est d'étudier la relation entre les altérations du langage et divers aspects cliniques et sociodémographiques chez un groupe de patients atteints de la maladie de Parkinson. Nous en avons sélectionné 46 , dont 42 présentent des altérations linguistiques de types variés liées à des variables sociodémographiques (âge, sexe et culture) et cliniques (période d'évolution de la maladie, échelle de Hoeh \& Yahr, UPDRS-III et dose de Ldopa). En fonction de la distribution des variables, le test $\mathrm{T}$ de Student ou le test U de Mann Whitney ont été utilisés.

MOTS CLÉS: linguistique clinique altérations linguistiques; maladie de Parkinson; variables cliniques; variables sociodémographiques.

SOMMAIRE: $1 . \quad$ Introduction. 2 Matériel et méthode. 3. Résultats. 4. Discussion. 5. Conclusions. 6. Limites de l'étude.
Fecha de Recepción Fecha de Revisión Fecha de Aceptación Fecha de Publicación
$05 / 02 / 2019$

$08 / 04 / 2019$

$10 / 05 / 2019$

$01 / 12 / 2020$ 


\title{
Alteraciones lingüisticas en la enfermedad de Parkinson. Aproximación estadística a un estudio con variantes.
}

\author{
MARÍa JESÚs PAREDEs DUARTE \& RAÚl EsPinosa Rosso
}

\section{INTRODUCCIÓN}

La enfermedad de Parkinson ha sido muy estudiada desde el punto de vista clínico tras su descubrimiento en el siglo XIX (Micheli, 2006) y posee una prevalencia elevada entre la población (13\% de la población mundial, en España entre 120.000 y 160.000 individuos, según la Federación Española de Parkinson), ya que se trata de una enfermedad frecuente, sobre todo en la senectud. En la actualidad, los estudios más desarrollados en este campo se centran en la detección temprana del párkinson y su posible prevención (Noyce et al., 2014, y Noyce et al., 2017), haciendo especial hincapié en las herramientas indispensables en su diagnóstico (Berardelli et al., 2013).

Por su parte, las alteraciones del lenguaje que presentan los enfermos de párkinson, aunque son consideradas como uno de los items evaluadores de los aspectos motores de la enfermedad, ya que se evalúan en la escala de motricidad Unified Parkinson's Disease Scale (UPDRS), junto a la expresión facial, el temblor, la rigidez, la postura, etc. (http://getm.sen.es/escalas-devaloracion/unified-parkinsons-disease-rating-scale-updrs/), han sido menos estudiadas, sobre todo desde un punto de vista estrictamente lingüístico. Aparecían como un apunte parcial en manuales y textos médicos y rara vez se veían analizadas por los logopedas.

Veamos algunos ejemplos recogidos en el trabajo de Sánchez Batista (2014). De la disartria hipocinética, alteración lingüística más frecuente y más citada por los autores, hablan Alberca Serrano, González Maldonado y Ochoa Amor (1996), Micheli (1998), Perea Bartolomé (1999), Love y Webb (2001), López del Val y Linazasoro Cristóbal (2004), Fiuza Asorey y Mayán Santos (2005), Webb y Adler (2008), Martínez-Sánchez (2010), López del Val, Linazaroso Cristóbal et al. (2011). La hipofonía y la voz monocorde aparecen comentadas en Alberca Serrano et al. (1996), Birkmayer y Danielczyk (1997), Perea Bartolomé (1999), Love y Webb (2001), Torres Carros et al. (2001), López del Val y Linazasoro Cristóbal (2004), Fiuza Asorey y Mayán Santos (2005), Webb y Adler (2008), Martínez-Sánchez (2010), López del Val, Linazasoro Cristóbal et al. (2011). Otros fenómenos de corte gramatical o léxico tienen menor tratamiento, como veremos después, en los resultados de este estudio.

Una perspectiva completa de cómo están afectados los diferentes componentes del lenguaje con su respectiva localización en las distintas zonas cerebrales se hacia inminentemente necesaria. Y más aún cuando ciertos trastornos del lenguaje pueden convertirse en diagnóstico y sintoma de la enfermedad (Melloni et al., 2015). 
En trabajos anteriores (Paredes Duarte y Espinosa Rosso, 2015), hemos ofrecido un panorama completo de las principales alteraciones del lenguaje de los enfermos de párkinson, clasificándolas, según herramientas básicas, por niveles lingüísticos, entendidos estos desde en la concepción más tradicional (Benveniste, 1997) de fonético-fonológico, gramatical, léxico, e incluso textual, "entendido el texto como la unidad máxima del nivel respectivo" (Cuenca, 2010) con la intervención de ciertas consideraciones pragmáticas. En este trabajo, pretendemos realizar un estudio estadístico de la relación entre estas alteraciones y determinados aspectos clínicos y sociodemográficos, estudio cuyos primeros resultados se mostraron en un póster de investigación en la XXXIV Reunión Anual de la Sociedad Andaluza de Neurología (Paredes Duarte, Espinosa Rosso y Sánchez Batista, 2016).

\section{MATERIAL Y MÉTODO}

\subsection{PARTICIPANTES}

Hemos partido de 46 pacientes afectados por la enfermedad de Parkinson, de los cuales, 42 presentaban alteraciones lingüisticas de diversa indole. Esos 46 pacientes forman parte de una muestra inicial de 50 pacientes que constituyó el corpus de partida de nuestras investigaciones y que fueron seleccionados de la Consulta de trastornos del movimiento del servicio de Neurología del Hospital Universitario Puerta del Mar de Cádiz.

La selección se hizo en virtud de una muestra de carácter estratificado o por cuotas, que nos pareció más representativa de los pacientes que acudian a dicha consulta y de los enfermos de párkinson en general que una muestra homogénea (Paredes Duarte y Espinosa Rosso, 2015) y que se basaba en criterios sociodemográficos como el sexo, la edad y el nivel cultural, variables que, en este trabajo, y junto a otras de carácter clínico, servirán para el estudio estadístico. Además, se desecharon pacientes que, bien por edad avanzada o por no padecer una enfermedad de Parkinson idiopática (que es el objeto de este estudio), problemas psiquiátricos asociados o la presentación de demencia, no aseguraban una relación estrecha entre las alteraciones lingüísticas y la enfermedad de Parkinson.

Para la investigación que ahora presentamos, la muestra se hizo más pequeña, suprimiendo a 4 pacientes de esos 50 iniciales porque desde el 2012 a la actualidad su enfermedad ha evolucionado mucho y se han quedado sumidos en el mutismo absoluto, por lo que ya no era posible analizar su conversación. Se distribuyeron los pacientes según variables sociodemográficas (sexo, edad, nivel cultural) y según variables clínicas (tiempo de evolución de la enfermedad, UPDRS, Hoehn \& Yahr y dosis de L-Dopa administrada).

En cuanto al sexo, la muestra no ha sido del todo equitativa -un número un poco más elevado de hombres que de mujeres-, pero sí representativa debido a que la enfermedad de Parkinson tiene una ligera predilección por 
los varones (García et al., 2008). Lo mismo ocurre con el nivel cultural, donde predominaba claramente el nivel medio, seguido del bajo, justificado porque es el perfil de enfermos que acuden a esta consulta, frente a una gran minoría de nivel alto ${ }^{1}$, aunque esto sí va a determinar conclusiones lingüísticas un poco forzadas, ya que algunas de las alteraciones pueden justificarse por una variante sociolingüistica de tipo diastrático, más que por la propia enfermedad de Parkinson. En lo que concierne a la edad, la mayoría de los pacientes se ubicó en el segundo grupo generacional (de 66 a 80 años), seguido de aquellos que se reparten entre los 81 y los 95 años, lo que resulta acorde con la edad de los pacientes afectados de Parkinson y, por tanto, los que acuden a esta consulta.

Por otro lado, entre los datos clínicos del paciente han sido incluidas cuatro variables médicas que según los estudios realizados hasta el momento (Paredes Duarte y Espinosa Rosso, 2015) y la comprobación empírica en enfermos concretos, van a influir directamente en el lenguaje de los enfermos de párkinson: 1) el tiempo de evolución del párkinson, 2) el estadio Hoehn y Yahr que se refiere a una escala para determinar en qué fase está el paciente (de la I a la V) en cuanto a la gravedad de su enfermedad ${ }^{2}, 3$ ) la escala UPDRS (Unified Parkinson Disease Rating Scale) que se utiliza en los estudios sobre la enfermedad de Parkinson para objetivar el estado funcional del paciente y que consta de cuatro partes siendo la tercera la que específicamente evalúa los aspectos motores (la puntuación máxima en la subescala III es de 68 puntos y cuanto más puntúe el paciente, peor estará desde el punto de vista motor) (Herrera, Cuetos y Ribacoba, 2012) y 4) la dosis de L-Dopa administrada.

En lo que respecta al tiempo de evolución de la enfermedad, aproximadamente la mitad de los pacientes están entre 1 y 5 años y la otra mitad se reparte entre los que están entre 5 y 10 años y 10 y 20 años, quedando casos excepcionales reservados para un tiempo de evolución de mayor de 20 años, lo que se justifica porque normalmente se pierde el seguimiento a estos pacientes de larga evolución ${ }^{3}$. En cuanto a la intensidad Hoehn y Yahr, la gran mayoría se reparten entre la escala II y III, siendo minoritarios los que se sitúan en la escala IV y V. Según la UPDRS, la muestra se reparte aproximadamente de modo equitativo entre los que están por debajo de 10, entre los que están entre 10 y 20 y los que tienen más de 20 . En cuanto a

\footnotetext{
${ }^{1}$ En lo que respecta al nivel cultural, hemos elegido la tricotomía alto/medio/bajo para generalizar y no especificar demasiado, lo que complicaria mucho los datos.

2 En la fase I los síntomas afectan solo a un hemicuerpo, en la fase II a los dos hemicuerpos, en la III hay alteraciones del equilibrio, caídas, etc. En la IV los pacientes precisan en algún momento del día el uso de silla de ruedas y en la fase $\mathrm{V}$ los pacientes son totalmente dependientes precisando uso de silla de ruedas la práctica totalidad del día.

${ }^{3}$ En cuanto a los pacientes de edad más avanzada, a los que se diagnostica más tarde, también van a desarrollar demencia antes y normalmente dejan de verse en la consulta porque se institucionalizan o porque están en una situación de inmovilidad tal que la familia ya no los trae. Además, estos pacientes toman dosis más bajas de L-Dopa puesto que no necesitan tanta medicación y, en general, tienen muy escasa respuesta a la misma, desarrollando frecuentemente complicaciones psiquiátricas.
} 
la dosis de L-Dopa administrada, la mayoría está entre 0 y 500 mg y muy pocos superan los $1000 \mathrm{mg}$ de L-Dopa.

\subsection{INSTRUMENTOS}

Para evaluar las posibles demencias y otros datos cognitivos, se utilizó el test mini-mental, concretamente el mini examen cognoscitivo (el MEC de Lobo) atendiendo a pruebas sobre la ubicación temporal, la orientación, la atención y el cálculo, la repetición y las operaciones aritméticas.

Como método de campo (Paredes Duarte y Espinosa Rosso, 2015) se utilizó "la entrevista individual que constituye la técnica de recogida de datos más conocida y más practicada en los estudios de habla (Hernández Campoy y Almeida, 2005: 135) y, dentro de esta, preferimos la entrevista anónima fugaz, que posee la ventaja de "solventar la paradoja del observador, por administrarse sin necesidad de grabación ni de que el informante sea consciente de estar siendo observado" (Hernández Campoy y Almedia, 2005: 139). Además, incluimos, siguiendo a Gallardo Paúls (2005) "la utilización del tema libre, que genera aún más espontaneidad en la entrevista".

Para ofrecer un estudio estadístico que nos llevara a un análisis descriptivo de partida utilizamos como método el Test T de Student y para realizar un análisis bivariante acudimos, según la distribución de las variables, de nuevo al Test T de Student, o al U de Mann Withney.

\subsection{PROCEDIMIENTO}

Una vez realizadas las entrevistas, tal como hemos comentado, de modo anónimo y fugaz, en el seno de la Consulta de trastornos de movimiento del hospital, se elaboraron, para la evaluación del lenguaje de los pacientes, unas plantillas que incluían, además de los citados datos sociodemográficos y clínicos, "la fecha de la consulta, el diagnóstico, la sintomatología y posibles incidencias generales en el lenguaje. También se incluyeron en la plantilla una gran enumeración de alteraciones lingüísticas ordenadas por niveles de análisis que respondian a los principales trastornos ofrecidos por los distintos estudiosos que citamos en la bibliografia y otras anomalias que incluimos fruto de la observación y la recogida de datos empíricos hecha en el seno de la citada consulta. Así contábamos con 5 tablas de alteraciones (articulatorias, prosódicas, gramaticales, léxicas y semánticas y textuales y pragmáticas) donde se enumeraban las diferentes anomalias lingüísticas" (Paredes Duarte y Espinosa Rosso, 2015).

El procesamiento de los datos nos llevó al recuento de las alteraciones lingüísticas más frecuentes que fueron sometidas, junto a las variables sociodemógráficas y clínicas, al método Test T de Student que nos ofreció los porcentajes en caso de las variables que no se pueden promediar, sexo y nivel cultural, y la desviación estándar (SD) en el resto (Paredes Duarte, Espinosa Rosso y Sánchez Batista, 2016): 


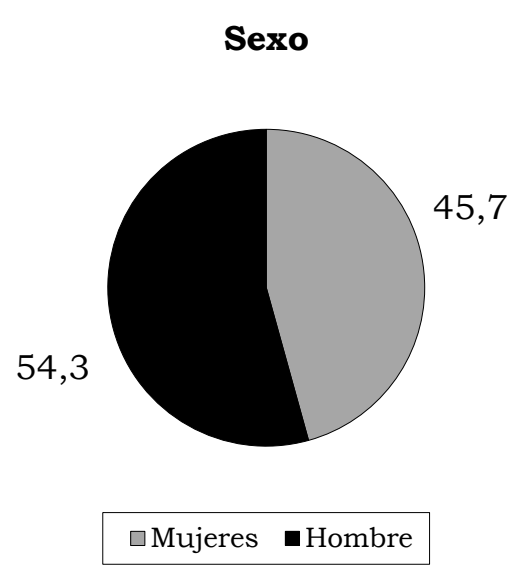

Ilustración 1: Porcentaje con la variable sexo

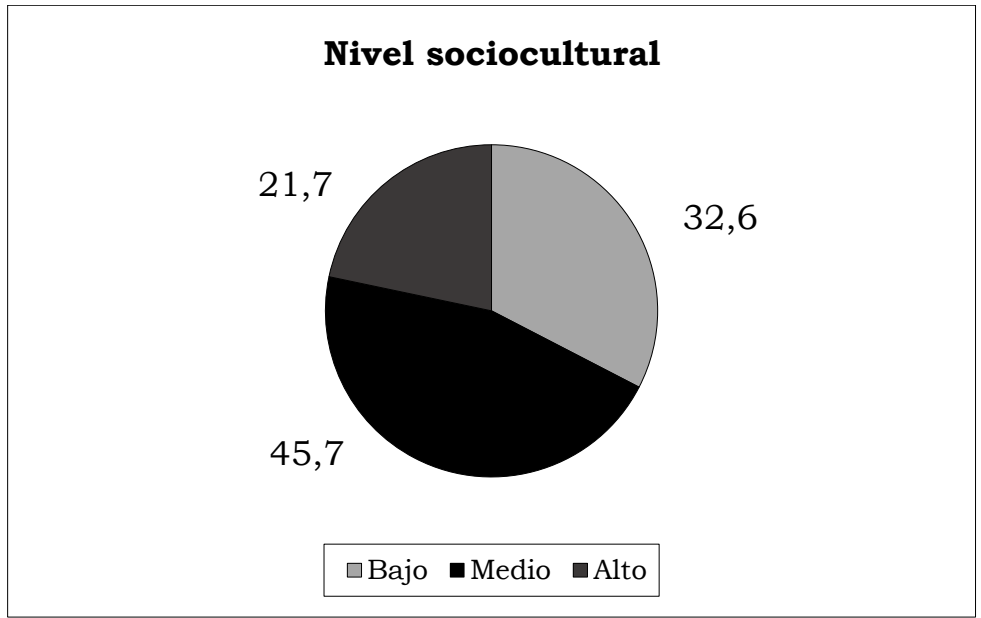

Ilustración 2: Porcentaje con la variable nivel sociocultural

\begin{tabular}{|l|c|c|}
\cline { 2 - 3 } \multicolumn{1}{c|}{} & MEDIA & $\begin{array}{c}\text { DESVIACIÓN } \\
\text { ESTADÍSTICA } \\
\text { ( }\end{array}$ \\
\hline EDAD & 72,41 & $\pm 10,41$ \\
\hline TIEMPO & 8,39 & $\pm 7,49$ \\
\hline UPDRS & 15,52 & $\pm 7,44$ \\
\hline HOEHN \& YAHR & 2,207 & $\pm 0,8204$ \\
\hline L-DOPA & 481,65 & $\pm 451,05$ \\
\hline
\end{tabular}

Ilustración 3: Desviación estándar con las variables: edad, tiempo de evolución de la enfermedad, UPDRS y Hoehn \& Yahr 
Una vez llegados a este punto, se realizó un análisis bivariante utilizando, según la distribución de las variables, el Test T de Student o U de Mann Withney para evaluar las posibles diferencias entre grupos, lo que nos llevó a los resultados que, a continuación, se enumeran.

Entre los problemas fonéticos que afectan al habla, el principal es la disartria hipocinética. Conocemos por disartria hipocinética (Darley, Aronson y Brown, 1969), ciertas alteraciones motrices que, como sintomas secundarios de la enfermedad de Parkinson, afectan a la musculatura de la respiración, la fonación, la resonancia, la articulación y la prosodia (entonación, melodía, ritmo). Todo ello afecta a la transmisión del mensaje con determinadas manifestaciones: 1) respiración superficial con irregularidades en el ciclo inhalación/exhalación, que produce alteraciones en el flujo de la voz y en la coordinación del inicio de la articulación con la fonación, produciéndose silencios inadecuados, 2) dificultad para realizar una abducción completa de los repliegues vocálicos con falta de flexibilidad y control de los movimientos laringeos (http://ciudadparkinson.do.am/news/parkinson_alteraciones_de_la_voz/2011-01-19-33), lo que justifica la voz temblorosa, ronca y el tono bajo y monótono con pérdida de volumen de la voz (hipofonía), 3) poca precisión articulatoria, sobre todo en palabras polisílabas y en largos discursos, quedando la articulación reducida a un farfulleo o murmullo que puede llevar a la inteligibilidad del mensaje, y titubeo ocasional al principio y con breves precipitaciones separadas por pausas, 4) la velocidad y estabilidad del ritmo del habla es variable, se tiende a acelerar el ritmo, por lo general se pierde el control sobre la articulación y se producen omisiones o sustituciones de sonidos y sílabas, a veces incluso con palilalia, es decir, repetición reiterada de frases o palabras y 5) la monotonía del tono y la intensidad de la voz, junto a una importante reducción de la acentuación y la entonación conllevan a una marcada disprosodia (no se transmite la intención comunicativa, por ejemplo, de pregunta, admiración, sorpresa, tristeza, etc.). Veamos algún ejemplo de ininteligibilidad, titubeo e incluso farfulleo (Paredes Duarte y Martín-Sánchez, 2018: 129):

$126((\mathrm{xx}))$ no me (he) da(d)o cuenta

138 en los ano(s) sesenta yyyyy tre(s) al sesenta y sei(s) / he trabajado en diferenteh sitio(s) $/ / / 4$

Por su parte, en el ámbito gramatical, se presentan alteraciones en las estructuras del sintagma y de la frase, por ejemplo (Paredes Duarte y Martín-Sánchez, 2018: 124):

2 ayer erannnnn ocho y media de la manana $\rightarrow((\mathrm{em}$ ee ee $\uparrow))$ fueron a recoherme

\footnotetext{
${ }^{4} \mathrm{El}$ simbolo $(\mathrm{XX})$ representa un fragmento indescifrable y la "yyy" reproduce un titubeo.
} 
con la furgoneta // llegamo(s) a San Fernando a lah nueve y media $\rightarrow 5$

También quedan muchos enunciados inconclusos (Paredes Duarte y Martín-Sánchez, 018: 124):

15 luego fui a casa de el yyy // y cene / ehthuve viendo la television aaa veee veee aproxi- aaaa ///(5.0) (queda en silencio sin completar la frase)

Provocando el anacoluto o ruptura de la frase con comienzo seguido de una nueva. Es el caso de (Paredes Duarte y Martín-Sánchez, 2018: 125):

43 el año pasa(d)o nos subimoh en el latigo $\rightarrow$ / yyy loћ llevaba pueћtho(s) aqui (se toca la garganta) // ehthaba que queee que me moria de miedo.

La repetición de subrutinas sintácticas y la ecolalia o repetición del final de un enunciado son también muy frecuentes (Paredes Duarte y MartínSánchez, 2018: 129):

134 no / porqueee hemo(s) ido a vario(s) sitio(s) / hemo(s) ehtha(d)o een ((hahthaloh diecii)) veinte ano(s) hemo(s) ehtha(d)o en Cordoba

Las principales alteraciones léxicas son: la anomia o dificultad para recuperar una palabra con su respectiva autocorrección por sustitución o por reformulación sintáctica y también la repetición léxica. Veamos un ejemplo de la primera (Paredes Duarte y Martín-Sánchez, 2018: 130)

189 (.eh?) ${ }^{\circ}$ azuca(r) le echa en uuuun le llena el hueco de azuca(r) le echa una poquita de agua $\rightarrow^{6}$

En lo que se refiere a la producción del discurso, uno de los rasgos más característicos es la dificultad para comenzarlo (veamos en el ejemplo 126, citado anteriormente, como el comienzo de la intervención está constituido por un fragmento indescifrable), algunas incoherencias textuales, excesivo uso de muletillas y algunos problemas de comprensión, como hemos dicho antes, sobre todo en lo que concierne a verbos de movimiento. En lo que respecta a la lecto-escritura, la micrografia se hace notable en estos pacientes (Paredes Duarte y Espinosa Rosso, 2015).

Por último, hay que señalar, en este apartado, el cumplimiento de las obligaciones éticas que supone un trabajo de este tipo ${ }^{7}$.

\footnotetext{
${ }^{5}$ La frase sin alteración sería algo así como "Ayer me recogieron con la furgoneta a las ocho y media de la mañana y llegamos a San Fernando a las nueve y media".

${ }^{6} \mathrm{El}$ "uuun" sustituye a la imposibilidad de recuperar la palabra que corresponde.

7 Con la aprobación del proyecto por parte del Comité ético del Hospital Puerta del Mar de Cádiz y a través de la firma de la Hoja del Consentimiento Informado (HIP) por parte de todos los pacientes sometidos al estudio o de sus familiares, si así lo requeria el caso. Todo ello en el
} 


\section{Resultados}

De los 42 pacientes estudiados, vimos que un $82,6 \%$ poseía anomalías fonéticas que afectaban al habla, el 54,3\% poseía alteraciones gramaticales y el mismo número presentaba problemas en la producción discursiva. Por último, en un $52,2 \%$ encontrábamos alteraciones léxicas. Veamos el gráfico:

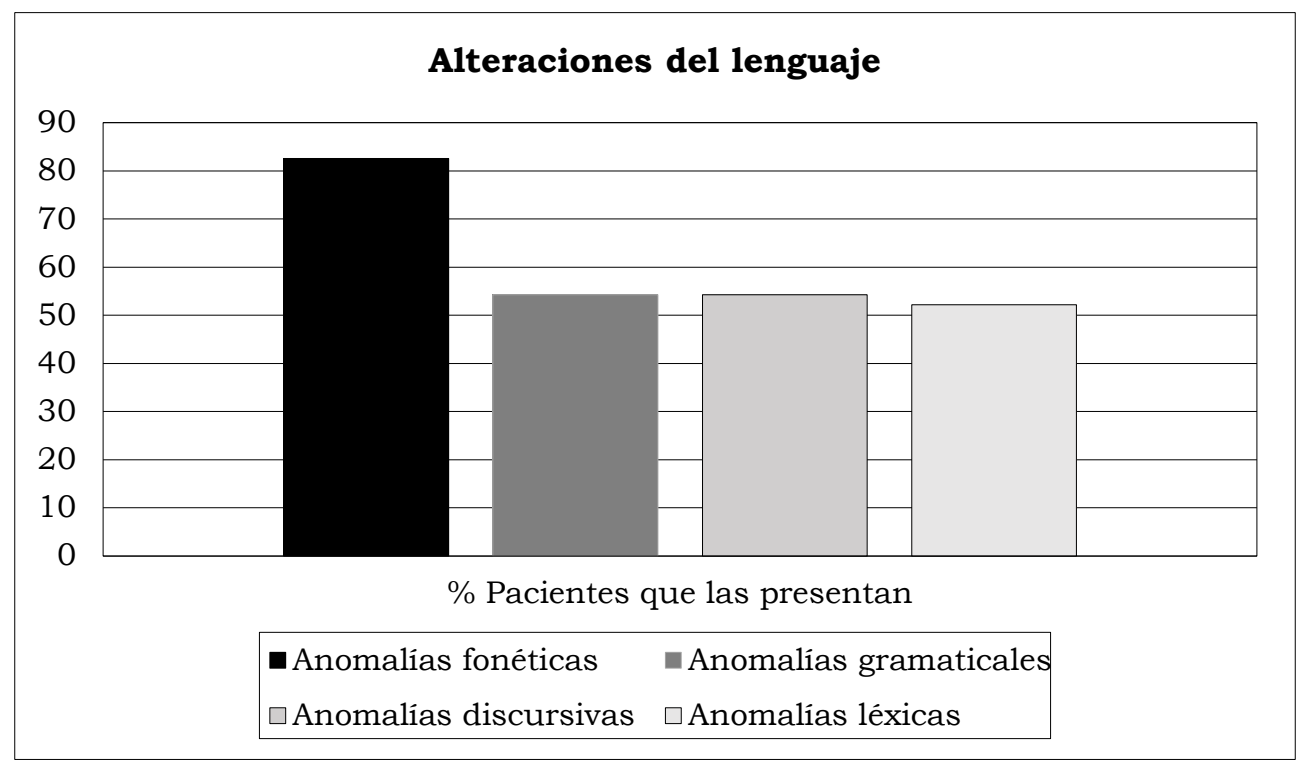

Ilustración 4: Porcentaje de pacientes que presentan los distintos grupos de alteraciones

Una vez realizado el análisis, encontramos diferencias estadísticamente significativas en el grupo de las alteraciones gramaticales cuando se relacionaban con el nivel sociocultural $(p=0.006)$, el tiempo de evolución de la enfermedad $(p=0.007)$, puntuación en las escalas Hoehn y Yahr $(p=0.029)$ y UPDRS-III ( $\mathrm{p}=0.012)$. Por su parte, entre las alteraciones que afectaban al discurso y las dosis de L-Dopa (Herrera, Cuetos y Ribacoba, 2012) se aprecia también relación $(p=0.06)$. Los demás parámetros analizados no arrojaron resultados significativos. 


\section{RELACIONES ENTRE LAS ALTERACIONES GRAMATICALES, VARIABLES SOCIOCULTURALES Y MÉDICAS}

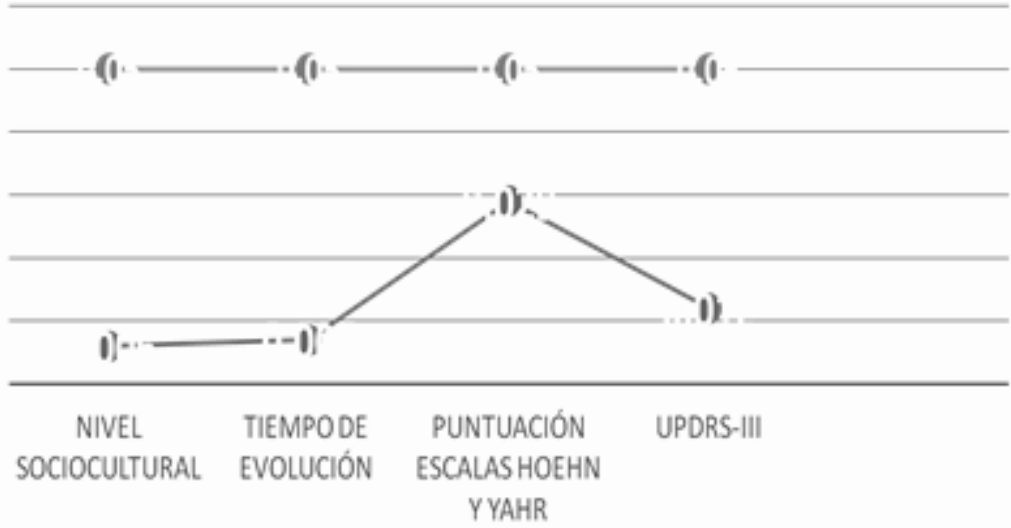

\section{-nivel de significación $=0,05 \quad$ p-value obtenido}

Ilustración 5: Relación entre las alteraciones gramaticales y variables socioculturales y médicas

\begin{tabular}{|c|c|}
\hline ALTERACIONES QUE AFECTAN AL DISCURSO & L-DOPA \\
\hline p-value & 0.06 \\
\hline
\end{tabular}

Ilustración 6: Relación entre las alteraciones que afectan al discurso y la dosis de L-Dopa administrada

\section{Discusión}

Tal como hemos citado en la introducción del trabajo, las alteraciones o problemas de indole fonética encontradas en los enfermos de Parkinson y que se derivan de lo que se conoce como disartria hipocinética, como por ejemplo voz monocorde, hipofonía, farfulleo, voz ronca, palilalia o disprosodia, son reseñados por muchos autores del panorama nacional e internacional. Sin embargo, otras alteraciones de carácter gramatical no han sido tan reseñadas, a pesar de que los problemas para construir frases gramaticalmente correctas han sido incluso noticia por ciertas investigaciones actuales (http://www.infosalus.com/actualidad/noticia-pbajos-parkinson-dana-capacidad-procesar-lenguaje-20110210142207.html). No encontramos autores que se dediquen al estudio de la alteración de estructuras sintagmáticas, inconclusión de frases, repetición y subrutinas sintácticas, ecolalia o anacoluto. 
En el campo de las alteraciones léxicas, ha sido estudiada la anomia (Allegri et al., 1992) y algunos problemas semánticos que atañen a comprensión de verbos que se asocian a la acción o al movimiento (Rodríguez Ferreiro y Cuetos, 2010). No se ha dicho nada, en el plano discursivo, de la incesante repetición de muletillas y muy poco (Fiuza Asorey y Mayán Santos, 2005), de una característica que es propia de los enfermos de Parkinson: la dificultad para comenzar un discurso que va estrechamente unida a lo que se conoce como imantación de la marcha. Tienen problemas para arrancar a hablar y a caminar, al principio van muy lentos y luego, en ambas actividades, cogen mucha velocidad.

Es, por tanto, en el plano del habla, donde principalmente se ubican los estudios de las alteraciones lingüísticas en enfermos de Parkinson, dejando a un lado otros fenómenos muy interesantes que corresponden a la lengua. Además, no se han hecho investigaciones como la que presentamos donde se tienen en cuenta variables sociodemográficas (edad, nivel sociocultural, sexo) y variables clínicas (tiempo de evolución de la enfermedad, dosis de Ldopa, UPDRS, Hoehn/Yahr). Cabe reseñar, por ejemplo, la diferencia lingüística existente entre dos personas de la misma edad, mismo sexo, con las mismas variables clínicas que poseen distinto nivel sociocultural. La importancia de esta variante sociolingüística podría explicar incluso casos atribuidos a condicionantes médicos como la hipercogenicidad de la sustancia negra (cf. Espinosa Rosso y Paredes Duarte, 2017).

\section{Conclusiones}

Como conclusiones al estudio, podemos señalar los siguientes parámetros:

- Existe una clara relación entre las alteraciones lingüísticas y el deterioro motor producido por la enfermedad de Parkinson, que apoya nuestra hipótesis de que las áreas que elaboran el lenguaje en la corteza cerebral están relacionadas con las estructuras subcorticales.

- Las alteraciones de indole gramatical, es decir aquellas que se refieren a las categorias morfológicas y sintácticas del lenguaje, son las que más resultados han arrojado, debido, en primer lugar, y como comentaremos en el apartado dedicado a las limitaciones del estudio, a que las fonéticofonológicas afectan a la gran mayoría de los pacientes sin distinguir variables. La distraria hipocinética caracteriza a la mayoría de los pacientes.

- Las estructuras morfológica y sintáctica del lenguaje se determinan por el nivel cultural, a mayor nivel cultural del paciente, menos afectadas estarán estas estructuras y por la escala de motricidad en la que se encuentren los pacientes, ya que el lenguaje es una actividad motora que 
no solo se justifica por una disartria a nivel fónico sino por una incapacidad de construir, concordar o utilizar sintagmas y oraciones simples o complejas.

- Las alteraciones léxicas no parecen afectadas por las variables estudiadas.

- Las alteraciones que se enmarcan en el discurso y que afectan a la conversación tienen que ver con la dosis de L-Dopa administrada a los pacientes, ya que esta sustancia justifica sobre todo la dificultad y lentitud al construir textos coherentes, sostener una conversación ordinaria o respetar los principios básicos comunicativos.

\section{LIMITACIONES DEL ESTUDIO}

Como ya se ha mencionado, la primera limitación ha sido el no poder establecer un análisis multivariante, como pretendiamos en primera instancia, debido a que entraban en juego un número demasiado elevado de alteraciones lingüisticas y no pocas variables de índole sociodemográfico y clínico. También hemos dicho que se intentó hacer el análisis por grupos de variables según los diferentes niveles lingüisticos, pero seguian siendo muchas variables en juego. Por ello se optó por un análisis bivariante mediante la metodología mencionada.

Por otra parte, al presentar la gran mayoría de pacientes de párkinson, anomalías de índole fonético-fonológica, no se mostraba variación cuando se incluían datos como la edad, el sexo, el nivel sociocultural, el tiempo de evolución de la enfermedad, las escalas de motricidad o la dosis de L-Dopa administrada. Quizás con una muestra superior en número se hubieran vislumbrado otros resultados.

También hemos hecho alusión, a circunstancias externas que no estratificaban la muestra de una manera exhaustiva, debido a la tipología demográfica del paciente que acude a la consulta del Hospital Universitario Puerta del Mar de Cádiz.

Por último, otras limitaciones que podrían citarse, ya desde el punto de vista lingüístico, sería el uso de la entrevista fugaz, que, aunque posee muchas ventajas frente a los test clásicos de evaluación, ya que el paciente no se siente observado, también presenta el problema del tiempo de duración de la conversación (no pueden observarse algunos fenómenos) y de la falta de documentos lingüísticos registrados (ya que no se realizaron grabaciones de ningún tipo).

Además de ampliarse, como ya hemos advertido, el número de pacientes estudiados caben destacar las siguientes investigaciones que se están llevando a cabo de modo interdisciplinar en el ámbito hospitalario acerca de la enfermedad de Parkinson y su incidencia en el lenguaje. Por un lado, se está realizando una tesis doctoral acerca del procesamiento lingüístico de los verbos de acción y movimiento en estos enfermos, que defiende una mayor dificultad en la comprensión de los mismos, relacionando de un modo muy 
interesante el lenguaje y el movimiento. Este estudio se ve completado por la aplicación de nuevas tecnologías que responden a aplicaciones para la rehabilitación del movimiento en estos pacientes (softwares como Virtualrehab o Temis, la herramienta Vedils), con el fin de demostrar, a través de la comparativa de grupos poblacionales afectados (un grupo de control, un grupo de parkinsonianos sometidos a las aplicaciones y un tercero no sometido) que la mejora de los aspectos motores conlleva una mejora de los aspectos lingüísticos alterados.

También, los autores de este trabajo presentaron al último Congreso de la Sociedad Española de Neurología (Valencia, 21-25 de noviembre de 2017), una investigación pionera que relaciona la hiperecogenicidad de la sustancia negra con determinados problemas lingüísticos en enfermos de Parkinson en estadio inicial, llegando a interesantísimas conclusiones. El estudio se ha llevado a cabo con 12 pacientes y, en la actualidad, se está ampliando a un número superior.

\section{REFERENCIAS}

\section{FUENTES DE INFORMACIÓN PRIMARIA}

ALBERCA SERRANO, R. et al. (1996): Diagnóstico y tratamiento de la enfermedad de Parkinson, Madrid: Ergon.

ALLEGRI, R. F. et al. (1992): "Evaluación neuropsicológica en la enfermedad de Parkinson", Medicina. 52, pp. 141-144.

BENVENISTE, E. (1997, 19a edic.): Problemas de lingüística general, Madrid: Siglo XXI.

BERARDELLI, A. et al., (2013): "EFNS/MDS-ES recommendations for the diagnosis of Parkinson's disease", European Journal of Neurology, 20, pp. 16-34.

BIRKMAYER, W. y DANIELCZYK, W. (1997): La enfermedad de Parkinson, Barcelona: Herder.

CUENCA, M. J. (2010): Gramática del texto, Madrid: Arco/Libros.

DARLEY, F. L.; ARONSON, A. E. \& BROWN, J. R. (1969): "Differential Diagnostic Patterns of Dysarthria", Journal of Speech, Language, and Hearing Research 12, pp. 246-269.

ESPINOSA ROSSO, R. y PAREDES DUARTE, M. J. (2017): "Relación entre sustancia negra y problemas lingüísticos en los enfermos de párkinson en estadio uno", LXIX Reunión Anual de la SEN (Valencia, 21-25 noviembre, 2017).

FIIUZA ASOREY, M. J. y MAYÁN SANTOS, J. M. (2005): ¿Qué es el Parkinson? Guía de tratamiento para el lenguaje, el habla y la voz, Madrid: Psicología Pirámide.

GALLARDO PAÚLS, B. y SANMARTÍN SÁEZ, J. (2005): Corpus PerLA. vol.1. Afasia fluente. Materiales para su estudio, Valencia: Universitat de València.

GARCÍA, S. et al. (2008): "Perspectiva histórica y aspectos epidemiológicos de la enfermedad de Parkinson", Med in Mex, 24 (1), pp. 2837.

HERNÁNDEZ CAMPOY, J. M. y ALMEIDA, M. (2005): Metodologia de la investigación sociolingüística, Granada: Editorial Comares.

HERRERA, E.; CUETOS, F. \& RIBACOBA, R. (2012): "Verbal fluency in Parkinson's disease patients on/off dopamine medication", Neuropsicologia, Dec 50 (14), pp. 3636-3640. 
LÓPEZ DE VAL, J. y LINAZASORO CRISTÓBAL, G. (2004): Los trastornos del movimiento, Madrid: Línea de Comunicación.

LÓPEZ DE VAL, J.; LINAZASORO CRISTÓBAL, G. et al. (2011): Parkinson y discinesias: Abordaje diagnóstico y terapéutico, Madrid: Editorial Médica Panamericana.

LOVE, R. J. y WEB, W. G. (2001): Neurología para los especialistas del habla y del lenguaje, Madrid: Editorial Médica Panamericana.

MARTÍNEZ-SÁNCHEZ, F. (2010): “Trastornos del habla y la voz en la enfermedad de Parkinson", Revista de Neurología, 51 (9), pp. 542550.

MELLONI, M. et al. (2015): "Cortical dynamics and subcortical signatures of motor-language coupling in Parkinson's disease", Scientific Reports, 5, 11899.

MICHELI, F. E. (1998): Enfermedad de Parkinson y trastornos relacionados, Buenos Aires: Editorial Médica Panamericana.

MICHELI, F. (2006, $2^{a}$ edic.): Enfermedad de Parkinson y trastornos asociados, Buenos Aires: Editorial Panamericana.

NOYCE, A. J. et al. (2014): "PREDICTPD: Identifyng risk of Parkinson's disease in the community: methods and baseline results", $\mathrm{J}$. Neurol Neurosurg Psychiatry, 85, pp. 31-37.

NOYCE, A. J. et al. (2017): "PREDICTPD: An online approach to prospectively identify risk indicators of Parkinson's disease", Mov. Disord., 16, pp. 219-226.

PAREDES DUARTE, M. J. (coord.) y MARTÍN-SÁNCHEZ, V. M. (ed.) et al. (cols.) (2018): Corpus de tras- tornos del lenguaje, Cádiz: Servicio de Publicaciones de la Universidad de Cádiz.

PAREDES DUARTE, M. J. y ESPINOSA ROSSO, R. (2015): "Lenguaje y parkinson: aproximación clínica", Dieguez-Vide, F. (ed.): Temas de lingüística clínica, Barcelona: Horsori, pp. 29-52.

PAREDES DUARTE, M. J.; ESPINOSA ROSSO, R. y SÁNCHEZ BATISTA, I. (2016): "Relación entre alteraciones del lenguaje en enfermedad de Parkinson y variables sociolingüísticas y clínicas", $X X X I X$ Reunión Anual de la Sociedad Andaluza de Neurología (Cádiz 6-7 de octubre de 2016).

PEREA BARTOLOMÉ, M. V. (1999): “Deterioro cognitivo en la enfermedad de Parkinson", First International Congress on Neuropsychology in Internet, Uniting horizons in Neuropsychology.

RODRÍGUEZ-FERREIRO, J. y CUETOS, F. (2010): "La comprensión de verbos en los enfermos de Parkinson", Ciencia Cognitiva, 4 (1), pp. 14-16. Disponible en: http://medina-psicologia.ugr.es/ cienciacognitiva/files/2010-1.pdf

SÁNCHEZ BATISTA, I. (2014): Trastornos motores y trastornos del lenguaje: una perspectiva desde la Lingüística clinica. Disponible en http://ro-

din.uca.es/xmlui/handle/ 10498 / 17587.

TORRES CARRROS, O. et al. (2001): "Rehabilitación del lenguaje en la enfermedad de Parkinson", Revista Mexicana de Neurociencia, 2 (4), pp. 241-244.

WEBB, W. G. \& ADLER, R. K. (2008): Neurology for the Speech-language Pathologist, Canada: Evolve Mosby Elsevier.

\section{WEBGRAFÍA}

Unified Parkinson's Disease Scale (UPDRS). (2 de diciembre 2017). Disponible en: http://getm.sen.es/escalas- de-valoracion/unifiedparkinsons-disease-ratingscale-updrs / (Fecha de consulta 30/09/2018). 
Descubren un camino para la detección precoz del parkinson. Disponible en: http://www.edicionesmedicas. com.ar/Actualidad/Ultimas_no ticias/Enfermedad_de_Parkins on9 (Fecha de consulta 30/11/2017).

El libro blanco del parkinson: aproximación análisis y propuesta de futuro. Disponible en: http://www.fedesparkinson.or g/libro_blanco.pdf (Fecha de consulta 30/09/2018).

Hallan sindromes del lenguaje que pueden ser un signo temprano del parkinson). Disponible en http://www.lanacion.com.ar/1 826159-hallan-deficits-del- lenguaje-que-pueden-ser-unsigno-temprano-del-parkinson (Fecha de consulta 02/12/2017).

Parkinson. Alteraciones de la voz. Disponible en http://ciudadparkinson.do.am/news/parkinson_alteraciones_de_la_voz/2011-01-19-33 (Fecha de consulta 30/09/2018).

Problemas para construir frases correctas. Disponible en http://www.infosalus.com/act ualidad/noticia-pbajosparkinson-dana-capacidadprocesar-lenguaje$20110210142207 . h t m l)$ (Fecha de consulta 30/09/2018). 